\section{Effect of Atmospheric Contaminants on Paper Chromatography of Urinary Indole and Phenol Acids}

SAMPLES of human urine have been examined for indole and phenol acids as a matter of routine in this laboratory for several months; the methods used for extraction and two-dimensional paper chromatography were described earlier ${ }^{1,2}$. The chromatograms were satisfactory until certain days in April, when the normally pink and purple spots given by indoles with $p$-dimethylaminobenzaldehyde showed a diminished colour intensity and brown discoloration. The colours became so weak in May that indole spots could not be seen with certainty. Unlike the indoles, the phenolic acids gave colours which were normal immediately after spraying with diazotized sulphanilic acid, but which faded and were discoloured afterwards to an increasing extent. No improvement was obtained when the components of the chromatographic solvents were purified extensively.

The occurrence of chromatographic difficulties appeared to coincide with an increased level of atmospheric contaminants. The eye-irritating haze known as 'smog', develops frequently in the Los Angeles area where topography and meteorological conditions favour the formation of a large stagnant air mass and the accumulation of fumes from numerous industries and other sources. 'Smog' contains ozone, organic peroxides, nitrogen oxides and other components'. The 'smog' level was low early in 1958 because of unusually heavy rains, but became noticeable in April and marked in May; an atmospheric ozone content of 0.35 parts per million was reported in Pasadena on May 15.

Several other features suggested that oxidative atmospheric contaminants were responsible for the destruction of indole spots on chromatograms. Ozone has been used preparatively to rupture the pyrrole ring of indole compounds ${ }^{4}$. The instability of ozone and organic peroxides in alkaline media is well known on the other hand, ozonization is frequently carried out in acetic acid. These properties may explain why one-dimensional chromatograms developed in isopropyl alcohol/ammonia/water $(8: 1: 1)$ furnished indole spots with almost normal colours with $p$-dimethylaminobenzaldehyde at a time when those run in benzene/propionic acid/water (100:70:5) did not.

A pertinent counterpart to these observations was provided by a report concerning the adverse effects of 'smog' on the response of plants to indoleacetic acid $^{5}$; the 'smog' was eliminated by filtering the air through charcoal ${ }^{6}$. This approach also afforded a solution to the present problem. Two sets of two-dimensional chromatograms were prepared from four different urine extracts. One set was dried in the hood as usual ; $p$-dimethylaminobenzaldehyde revealed faint brown (instead of blue) spots of only 5-hydroxyindoleacetic acid. The second set was dried in empty chromatography jars, after each solvent and after spraying, by a current of air which had passed through a 2-l. aspirator bottle filled with $8-12$ mesh activated charcoal ; 14 indole spots with normal colours were observed on this set of chromatograms. A charcoal unit large enough to ensure a 'smog'-free room is being designed.

The formation of 'smog' is a problem of increasing importance in densely populated and heavily industrialized cities. These findings, therefore, may be of interest to investigators working in such areas with small amounts of compounds or biological systems which are sensitive to the action of atmospheric contaminants.

We wish to thank Dr. A. J. Haagen-Smit and Dr. F. W. Went for helpful suggestions. This study was supported by a grant from the Ford Foundation.

\section{K. N. F. SHAW}

J. Trevarthen

Gates and Crellin Laboratories of Chemistry,

California Institute of Technology, Pasadena, California. July 1 .

${ }^{1}$ Armstrong, M. D. Shaw, K. N. F., Gortatowski, M. G., and Singer, H., J. Biol. Chem., 232, 17 (1958).

${ }^{2}$ Armstrong, M. D., Shaw, K. N. F., and Wall, P. F., J. Biol. Chem. 218,293 (1956)

${ }^{3}$ Haagen-Smit, A. J., Indust. Eng. Chem., 48, 65A (1956).

${ }^{4}$ Witkop, B., Ann. Chem., 556, 103 (1944).

${ }^{5}$ Hull, H. M., Went, F. W., and Yamada, X., Plant Physiol., 29, 18.2 (1954).

"Went, F. W., "The Experimental C'ontrol of Plant Growth", 51 (Chronica Botanica Co., Waltham, Mass., 1957).

\section{Inhibitory Action of Ethyl Mercaptan on Intracellular Tubercle Bacilli}

We have previously described the anti-tuberculous activity in unice of ethyl mercaptan and its derivatives ${ }^{1,2}$. The marked activity which was observed did not seem to correspond with the slight bacteriostatic action of ethyl mereaptan against Mycobacterium tuberculosis in vitro. Since the bacilli in tuberculosis are intracellular, we decided to test the effect of ethyl mercaptan against bacilli so located. For this purpose we used the method described by Suter $^{3}$ in which tubercle bacilli are grown in vitro within monocytes derived from peritoneal exudates of normal guinea pigs. Because of the high volatility of ethyl mercaptan it was necessary to modify Suter's method of incubating the cover-slips on which the monocytes and bacteria were attached. The tubes containing the cover-slips were sealed by heat before they were incubated, at an angle of $30^{\circ}$ from the vertical, without rotation. This modification still allowed good growth of the bacteria and isoniazid was highly effective (Table 1).

Four cover-slip preparations from each treatment were stained at intervals of $1,3,5$ and 7 days and 200 monocytes were examined on each slide and classified as containing $0,1-10,11-20$, and more than 20 bacteria respectively. The activity of an inhibitory compound can be seen most conveniently from the percentage of cells containing more than 20 bacteria in the 7-day cultures.

Ethyl mercaptan was inhibitory at concentrations of the order of $10 \mu \mathrm{gm} . / \mathrm{ml}$., methyl mercaptan was ineffective ; indeed it partially antagonized the effect of ethyl mercaptan. This is in accordance with our observations in mice ${ }^{2}$. The specificity of this effect was demonstrated by the failure of ethyl mercaptan to inhibit the intracellular growth of a strain of bacilli which had been made resistant to treatment with thiol esters in mice $^{2}$ (Table 1). Several potential metabolites of ethyl mercaptan, including methyl ethyl sulphide, methyl ethyl sulphone, $\beta$-hydroxyethyl methyl sulphide and $\beta$-hydroxyethyl methylsulphone were inactive, whereas the diethyl disulphide was active. 